\title{
Quality difference study of six varieties of Ganoderma lucidum with different origins
}

\author{
Juan Lu ${ }^{1}$, Jia-Zhang Qin ${ }^{2}$, Ping Chen ${ }^{2}{ }^{*}$, Xi Chen ${ }^{1}$, Ying-Zhi Zhang ${ }^{1}$ and Si-Jia Zhao ${ }^{3}$ \\ ${ }^{1}$ Institute of Medicinal Plant Development, Chinese Academy of Medical Sciences and Peking Union Medical College, Beijing, China \\ ${ }^{2}$ Beijing Yikangtang Medical Research Institute, Beijing, China \\ ${ }^{3}$ Beijing Hengjitang Medical Technology Development Co., Ltd., Beijing, China
}

\section{Edited by:}

Xiao-Ling Zhu, Peking University

Health Science Center, China

Reviewed by:

Yili Yang, Second Military Medical

University, China

Maria Do Céu Gonçalves Da Costa Laboratório Nacional de Energia e Geologia, Portugal

Maria Camilla Bergonzi, University of

Florence, Italy

\section{*Correspondence:}

Juan Lu, Institute of Medicinal Plant

Development, Chinese Academy of

Medical Sciences and Peking Union

Medical College, Beijing 100193,

China.

e-mail: jlu@implad.ac.cn

Ping Chen, Beijing Hengjitang

Medical Technology Development

Co., Ltd., Beijing 100070, China.

e-mail:cp@h5050.com
The quality difference of six varieties Ganoderma lucidum with different origins was investigated in this study by comparing the contents of ganoderic acid $A$ and $B$, polysaccharide, and triterpenoids. The contents of ganoderic acid $A$ and $B$ in $G$. lucidum were analyzed by ultra performance liquid chromatography (UPLC). There was higher content of ganoderic acid $A$ in G. lucidum of Dabie Mountain and Longquan. The G. lucidum from Longquan has the highest content of ganoderic acid $\mathrm{B}$. The content of polysaccharide was determined by Anthrone-sulfuric acid method. The highest of polysaccharide content is G. lucidum from Liaocheng. The content of triterpenoid in G. Iucidum was quantified by ultraviolet spectrophotometer at $548.1 \mathrm{~nm}$ using Ursolic acid as standard. The G. Iucidum from Dabie Mountain has the highest content of triterpenoids. In summary, the content of ganoderic acid $A$ and $B$, polysaccharide, and triterpenoids in G. lucidum with different origins are remarkably different, which may be caused by the conditions of cultivation and geographic environment.

Keywords: Ganoderma lucidum, ganoderic acid A, ganoderic acid B, polysaccharide, triterpenoids, content determination

\section{INTRODUCTION}

Ganoderma lucidum Karst a medicinal fungus, belonging to Basidiomycetes, Aphyllophorales, Ganodermataceae, is widely used in Oriental medicine to maintain health. With both edible and medicinal value, it has more than 2000 years of history in China. And the annual output of G. lucidum is over 10000 tons. G. lucidum has the function of anti-aging, enhancing immunity, radioprotective, and liver detoxification as well as inhibiting malignant tumor growth (Zhao et al., 1999; Lin, 2007; Lü et al., 2011). The chemical composition of G. lucidum is complex, which contains 11 categories of active substances, such as polysaccharides, triterpenoids, fats and oils, organic germanium, inorganic ions, and sterols. These ingredients are closely related to their pharmacological activity (el-Mekkawy et al., 2007). Polysaccharides and triterpenoids are considered to be its main medicinal components (Wang and Sun, 1990; Zhao et al., 2002; Lin, 2007). The quality of G. lucidum is evaluated though the content of polysaccharide in "Chinese Pharmacopeia," but Ganoderic acid in Japan (Zhang and Yang, 2006). Ganoderic acid belongs to triterpenoids, which has a wide range of pharmacological active components. It has become a hot study subject in G. lucidum (Chen and Yu, 1990; Yang et al., 1995; Zhou et al., 2004). Ganoderic acid A and B content account for more than half of G. lucidum (Ding et al., 2009), so the determination of ganoderic acid A and B content can be used as the scientific basis for judging quality of G. lucidum.

Because wild fungus resources are limited and artificial cultivation of G. lucidum is affected by origin, cultivation, harvesting conditions, and so on. These factors lead to different quality productions of G. lucidum. We tested the G. lucidum samples from some main producing areas in Shandong Liaocheng, Jiangsu Nantong, Fujian Wuyi Mountain Zhejiang Longquan, Jilin Changbai Mountain, and Anhui Dabie Mountain. The test is focused on the contents of polysaccharide, triterpenoid, and ganoderic acid A and B. The result is to provide the basis of procurement for using $G$. lucidum as main raw materials.

\section{MATERIALS AND METHODS MATERIALS}

Ganoderma lucidum karst is used for this experiment which is respectively from the Shandong Liaocheng, Jiangsu Nantong, Fujian Wuyi Mountain, Zhejiang Longquan, Jilin Changbai Mountain, and Anhui Dabie Mountain. In addition to G. lucidum from Shandong Liaocheng cultured on cotton seed, others are wood cultured.

\section{INSTRUMENTS AND REAGENT}

Waters Acquity ultra performance liquid chromatography (UPLC), KQ-250E type ultrasonic cleaner (Kunshan Ultrasonic Instrument Co., Ltd.), AB265-S/100000 electronic balance (Mettler Toledo). TU-1900 type ultraviolet-visible spectrophotometer (Beijing Purkinje General Instrument Co., Ltd.). Standard ganoderic acid A, ganoderic acid B (purity $>98 \%$ ) provided by Shanghai Tong Tian Biotechnology Co., Ltd. Urolic acid reference substance provided by the national institute for the control of 
pharmaceutical and biological products. Acetonitrile (chromatographic grade), double distilled water, phosphoric acid, chloroform, petroleum ether, ethyl acetate, methanol, and other reagents are all analytically pure.

\section{METHODS}

\section{Ganoderic acid A and ganoderic acid B content determination}

Chromatographic conditions. The chromatographic column was Waters X-BridgeC18 $(4.6 \mathrm{~mm} \times 150 \mathrm{~mm}, 3.5 \mu \mathrm{m})$; the detection wavelength was set at $252 \mathrm{~nm}$; gradient elution, liquid phase gradient ratio, and time relationships as shown in Table 1; the column temperature was kept at $40^{\circ} \mathrm{C}$, and the flow rate was $0.4 \mathrm{ml} / \mathrm{min}$; the injection volume was $10 \mu \mathrm{l}$. In these chromatographic conditions, ganoderic acid A and ganoderic acid B Mixed reference substance, and G. lucidum extraction UPLC spectrum diagram as shown in Figure 1.

\section{PREPARATION OF STANDARD SOLUTION AND CALIBRATION CURVES}

Precisely weighing amount of each reference substance and then put it into a $10-\mathrm{ml}$ volumetric flask respectively, adding methanol to dissolve and to the constant volume, reaching concentrations

Table 1 | Effect of different time of elution gradient ratio.

\begin{tabular}{lll}
\hline Time (min) & Acetonitrile & $\mathbf{0 . 0 3} \%$ phosphoric acid aqueous solution \\
\hline 0 & 30 & 70 \\
5 & 30 & 70 \\
50 & 35 & 65 \\
60 & 60 & 40
\end{tabular}

of $1.802 \mathrm{mg} / \mathrm{ml}$ of ganoderic acid A and $1.020 \mathrm{mg} / \mathrm{ml}$ of ganoderic acid B. Precisely weigh the liquid reserves each $8.0 \mathrm{ml}$ in $25 \mathrm{ml}$ volumetric flask, add methanol to scale, and shake to make mixed standard stock solution of ganoderic acid A and ganoderic acid B whose concentration are 0.577 and $0.326 \mathrm{mg} / \mathrm{ml}$. Precisely weigh standard stock solution $2.0-10 \mathrm{ml}$ volumetric flask, dilute to the mark with methanol, and then shake to get mixed standard solution of ganoderic acid $\mathrm{A}$ and $\mathrm{B}$ whose mass concentration are 0.100 and $0.065 \mathrm{mg} / \mathrm{ml}$.

According to the method of Zhao et al. (2009), precisely measure mixed control solution $0.5,1,2,4,6,8.0$ to $10 \mathrm{ml}$ volumetric flask, dilute to the mark with methanol, and shake to get a series of standard solution. Respectively take a $10-\mu l$ sample of the mixed standard solution to analyze under the chromatographic conditions. Draw a standard curve and make regression calculation with mass concentration of the reference as abscissa, peak area as the ordinate, the results show that, the regression equation of ganoderic acid $\mathrm{A}$ is $Y=1.9 \mathrm{E}+07 \mathrm{X}-122000$ and ganoderic acid $\mathrm{B}$ is $R^{2}=0.9996 ; Y=2.0 \mathrm{E}+07 \mathrm{X}+139583, R^{2}=0.9991$. Ganoderic acid A and ganoderic acid B respectively in 28.85-400.8, $16.30-260.8 \mu \mathrm{g} / \mathrm{ml}$ are in good linear relation.

\section{Preparation of sample solution}

According to the method of Liu (2008), the accurately weighed powder sample $(250 \mathrm{mg}$ ) was extracted with $100 \mathrm{ml}$ chloroform by the heating reflux for $1 \mathrm{~h}$. The extract was filtered with filter paper which washed by methanol. After evaporating chloroform to dryness by a rotary evaporator, residue was dissolved in methanol in a 5 -ml flask, and then filtered through a $0.45-\mu \mathrm{m}$ membrane. Ten microliters of sample solution were injected into the UPLC system for analyzing.
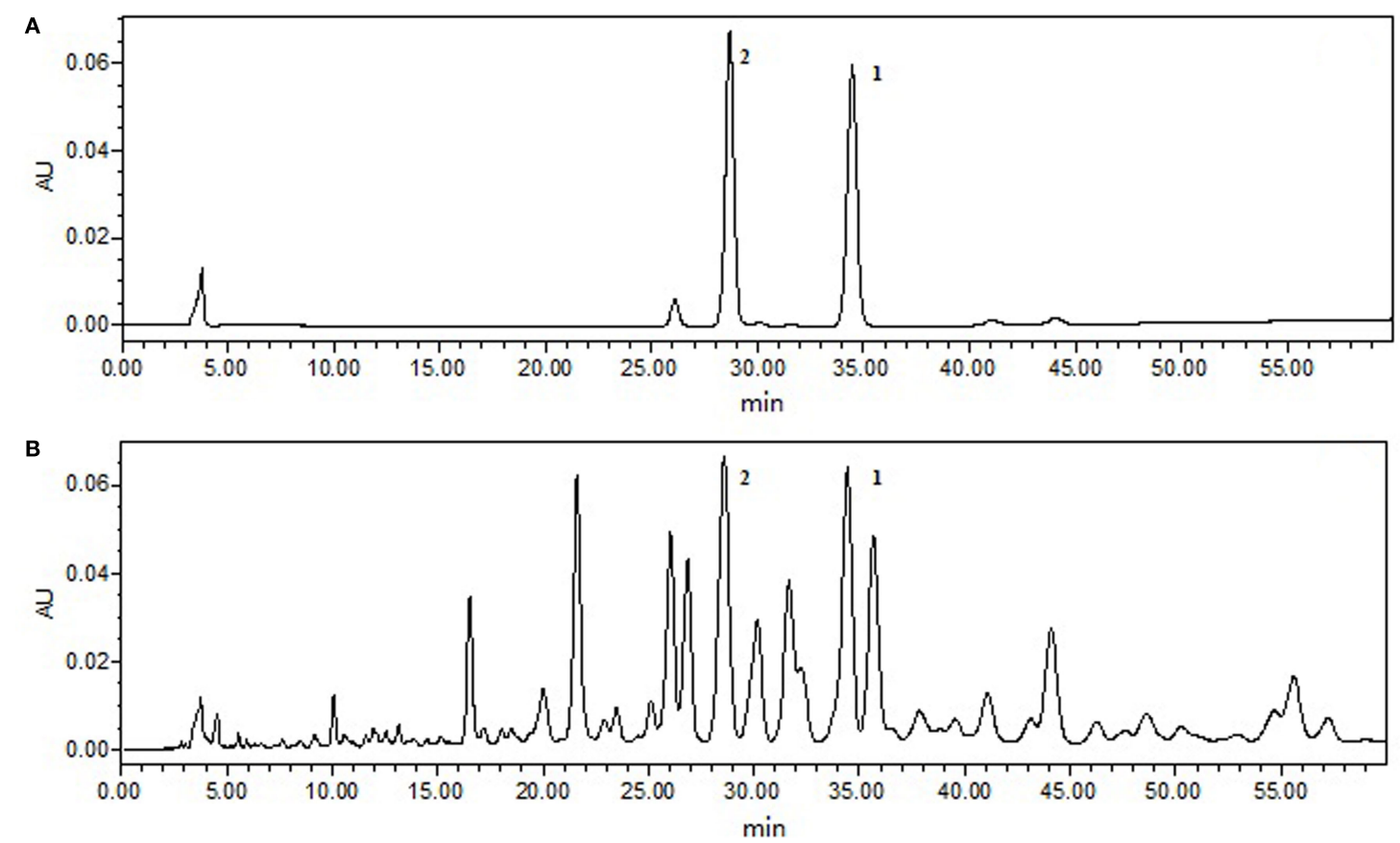

FIGURE 1 | Mixed reference substance (A) and Ganoderma lucidum extraction (B) UPLC spectra. 


\section{Precision test}

According to the chromatographic conditions, taking ganoderic acid $A$ and ganoderic acid $B$ mixed reference solution to successive injection six time, and recording the peak area. The RSD of peak area of ganoderic acid A and ganoderic acid B was 1.6 and 2.4\% respectively.

\section{Repetitive test}

Accurately weighed G. lucidum samples of six from Dabie Mountain, according to methods of above, the RSD of contents of ganoderic acid $\mathrm{A}$ and ganoderic acid $\mathrm{B}$ is 3.1 and $1.8 \%$ respectively. The result shows that this method has a good repetitiveness.

\section{Stability experiment}

Take sample solution from Anhui Dabie Mountain for the test, which is in $0,2,4,6,8,10,12,24 \mathrm{~h}$ at room temperature and record peak area. The results show that the sample solution has good stability in $24 \mathrm{~h}$ and the RSD of peak area of ganoderic acid $\mathrm{A}$ and ganoderic acid B were 0.9 and $1.4 \%$.

\section{Recovery rate test}

Take nine portions of G. lucidum $(0.25 \mathrm{~g})$ from the Wuyishan which the content of ganoderic acid $\mathrm{A}$ and ganoderic acid $\mathrm{B}$ are known. Divide the portions into three groups and add the control solution of low, middle, high concentrations of ganoderic acid A and ganoderic acid B to each portion, then calculate the recovery rate follow the method above. The result is as shown in Table 2.

\section{Determination of polysaccharide}

Sample preparation. Accurately weighing $2.0 \mathrm{~g}$ of power sample, extracted by Soxhlet extractor with $90 \mathrm{ml}$ water in the roundbottom flask, and heated under reflux for $6 \mathrm{~h}$, then transfer the

Table 2 |The recovery rate test $(n=3)$.

\begin{tabular}{|c|c|c|c|c|c|}
\hline Composition & Label & $\begin{array}{l}\text { Sample } \\
\text { quantity } \\
\text { (mg) }\end{array}$ & $\begin{array}{l}\text { Adding } \\
\text { amount } \\
\text { (mg) }\end{array}$ & $\begin{array}{l}\text { Recovery } \\
\text { rate }(\%)\end{array}$ & RSD (\%) \\
\hline \multirow[t]{9}{*}{ Acid A } & 1 & 0.5385 & 0.2481 & 95.4 & 1.41 \\
\hline & 2 & 0.5293 & 0.2417 & 97.6 & \\
\hline & 3 & 0.5236 & 0.2352 & 94.4 & \\
\hline & 4 & 0.5427 & 0.5228 & 97.7 & 1.02 \\
\hline & 5 & 0.5328 & 0.5173 & 95.9 & \\
\hline & 6 & 0.5343 & 0.5284 & 98.6 & \\
\hline & 7 & 0.5422 & 0.7362 & 98.1 & 1.28 \\
\hline & 8 & 0.5375 & 0.7340 & 100.1 & \\
\hline & 9 & 0.5432 & 0.7274 & 99.4 & \\
\hline \multirow[t]{9}{*}{ Acid B } & 1 & 0.1745 & 0.0936 & 93.7 & 1.28 \\
\hline & 2 & 0.1715 & 0.0915 & 94.2 & \\
\hline & 3 & 0.1697 & 0.0867 & 96.0 & \\
\hline & 4 & 0.1759 & 0.1864 & 95.3 & 1.75 \\
\hline & 5 & 0.1726 & 0.1735 & 96.9 & \\
\hline & 6 & 0.1731 & 0.1726 & 98.7 & \\
\hline & 7 & 0.1757 & 0.2619 & 97.1 & 1.19 \\
\hline & 8 & 0.1742 & 0.2700 & 98.6 & \\
\hline & 9 & 0.176 & 0.2623 & 99.4 & \\
\hline
\end{tabular}

extract to a $100 \mathrm{ml}$ flask, add water to the scale. Precisely measured $10 \mathrm{ml}$ extract, added ethanol $150 \mathrm{ml}$, placed for $12 \mathrm{~h}$ at $4^{\circ} \mathrm{C}$. The extract separated by centrifugal precipitation, the precipitate is dissolve in water in a $50-\mathrm{ml}$ flask as the sample solution.

Preparation of standard curve. D-Glucose anhydrous ( $25 \mathrm{mg}$ ) is accurately weighed and then dissolved in $25 \mathrm{ml}$ of double distilled water, $1 \mathrm{ml}$ solution is drawn to dilute 100 times with double distilled water to produce corresponding stock standard solution $(0.01 \mathrm{mg} / \mathrm{ml})$. Accurately draw glucose control solution 0.2 , $0.4,0.6,0.8,1,1.2 \mathrm{ml}$ to the $10 \mathrm{ml}$ test tube, add water to the volume of $2.0 \mathrm{ml}$, precisely add anthrone-sulfuric acid [ $1.0 \mathrm{~g}$ of anthrone was dissolved in sulfuric acid (80\%) in a $100 \mathrm{ml}$ flask] $6 \mathrm{ml}$, heated for $15 \mathrm{~min}$, then remove and put in ice-water to cool for $15 \mathrm{~min}$, with the corresponding reagent as control. Determine the absorbance in the $625 \mathrm{~nm}$ wavelength and make it as the ordinate, concentration as abscissa to establish a standard curve.

Precisely measure the sample solution $2 \mathrm{ml}$, put it into $10 \mathrm{ml}$ test tube, Follow the method of establishing the standard curve, as the "precisely add anthrone-sulfuric acid $6 \mathrm{ml}$ " begin to determine absorbance. Then calculate the content of the polysaccharide according to the standard curve.

\section{Triterpenoid determination}

Preparation of standard curve. Accurately weigh $1.15 \mathrm{mg}$ of the ursolic acid, dissolve in $10 \mathrm{ml}$ ethyl acetate to produce corresponding stock standard solution. Take $0,0.10,0.20,0.40,0.60$, $0.80,1$, and $1.20 \mathrm{ml}$ control solution to dryness in a water bath at $100^{\circ} \mathrm{C}$. Then add $0.40 \mathrm{ml} 5 \%$ vanillin-acetic acid solution and $1 \mathrm{ml}$ perchloric acid, at $60^{\circ} \mathrm{C}$ water bath heating for $15 \mathrm{~min}$ then move it into ice-water bath, add $5 \mathrm{ml}$ acetic acid, place it at room temperature for $15 \mathrm{~min}$. Determine its absorbance in the $548.1 \mathrm{~nm}$.

Draw standard curve based on the determination result. Standard weight in $0-0.14 \mathrm{mg}$ range showed a good linear relationship with the absorbance value, the linear regression equation was $Y=0.2158 \mathrm{X}-0.0018$, correlation coefficient $r=0.9991$.

Extraction of triterpenoids. Triterpenoid extracts were prepared by $95 \%$ alcohol extraction as described before (Hou and Liu, 2010). Accurately weigh $200 \mathrm{~g}$ of dry G. lucidum powder for extraction. Take G. lucidum extracts about $10 \mathrm{mg}$ to dissolve in $10 \mathrm{ml}$ ethyl acetate, and determine its absorbance following the method above.

\section{RESULTS}

\section{CONTENT DETERMINATION OF DIFFERENT ORIGIN OF GANODERIC} ACID A AND GANODERIC ACID B

The content of ganoderic acid A and ganoderic acid B is as shown in Table 3. The content of ganoderic acid A of Dabie Mountain is the highest $(7.254 \mathrm{mg} / \mathrm{g})$; the followed behind is Longquan (6.658 mg/g), Shandong (1.959 mg/g). Ganoderic acid B content for Longquan $(4.574 \mathrm{mg} / \mathrm{g})$ is the highest.

\section{CONTENT DETERMINATION OF POLYSACCHARIDE}

The content of polysaccharide has significant differences. The highest content of G. lucidum polysaccharides is in Shandong, followed by Wuyi Mountain (7.38\%); the lowest (1.85\%) is in Dabie Mountain (see Table 4). 
Table 3 | Six kinds of ganoderic acid A and ganoderic acid B content.

\begin{tabular}{lllllll}
\hline Category & $\begin{array}{l}\text { Dabie mountain } \\
(\mathbf{m g} / \mathbf{g})\end{array}$ & $\begin{array}{l}\text { Longquan } \\
\mathbf{( m g / g )}\end{array}$ & $\begin{array}{l}\text { Nantong } \\
(\mathbf{m g} / \mathbf{g})\end{array}$ & $\begin{array}{l}\text { Changbai mountain } \\
\mathbf{( m g / g )}\end{array}$ & $\begin{array}{l}\text { Wuyi mountain } \\
(\mathbf{m g} / \mathbf{g})\end{array}$ & $\begin{array}{l}\text { Liaocheng } \\
(\mathbf{m g} / \mathbf{g})\end{array}$ \\
\hline Ganoderic acid A & 7.254 & 6.658 & 3.563 & 2.618 & 2.154 \\
Ganoderic acid B & 0.337 & 4.574 & 0.574 & 1.450 & 0.698 \\
\hline
\end{tabular}

Table 4 | Polysaccharide content of Ganoderma lucidum in different regions.

\begin{tabular}{ll}
\hline Sample & Mass fraction (\%) \\
\hline Dabie mountain & 1.85 \\
Longquan & 5.88 \\
Nantong & 3.30 \\
Changbai mountain & 6.03 \\
Wuyi mountain & 7.38 \\
Liaocheng & 9.08 \\
The SDs & 2.64 \\
\hline
\end{tabular}

\section{CONTENT DETERMINATION OF TRITERPENOID}

The content of triterpenoid from different origins has been shown in Table 5. The highest content of triterpenoid of G. lucidum is cultivated in Dabie mountain (5.38\%), the lowest is in Longquan $(2.07 \%)$. The difference between them is significant.

\section{DISCUSSION}

Triterpenoid and polysaccharide are as the basis of quality of Ganoderma product. Triterpenoid has significant effect in immune regulation and antitumor (Morigiwa et al., 1986; Ceng and Bao, 2004; Huang and Xiao, 2008); its content decides the antitumor effect of G. lucidum products. Polysaccharide has the function of improving immunity, antitumor effects, removing free radical, hypoglycemic, lipid-lowering ( $\mathrm{Xu}$ and $\mathrm{Xu}, 2003$ ), and other functions. In recent years, the study has attracted many researchers (Lin et al., 2002; Cao and Lin, 2004). Ganoderic acid $B$ and lucidenic acid $A$ have the inhibitory activity against HIV-1 protease (Min et al., 1998). For the reason of above, we got the main medical ingredients of G. lucidum from six origins.

Through this experimental data, both the triterpenoid and ganoderic acid B are the highest in G. lucidum from Dabie mountain. But the highest polysaccharide of artificial Cultivation $G$. lucidum is from Liaocheng. There are several reasons impacting the accumulation of polysaccharides and triterpenoids. First, the same species of G. lucidum from different origins due to the culture medium, the growth environment, different stages of growth, and covered soil or not, will have different polysaccharide content (Li et al., 1997; Ding et al., 1999; Wei et al., 2006; Chen et al., 2009; Ye et al., 2010). Second, the content of polysaccharide and

\section{REFERENCES}

Cao, Q. J., and Lin, Z. B. (2004). Antitumor and anti-angiogenic activity of Ganoderma lucidum polysaccharides peptide. Acta Pharmacol. Sin. 25, 833-838. Ceng, X. L., and Bao, H. Y. (2004). Advances of researches
Table 5 | Triterpenoid content of Ganoderma lucidum in different regions.

\begin{tabular}{ll}
\hline Sample & Mass fraction (\%) \\
\hline Dabie mountain & 5.38 \\
Longquan & 2.07 \\
Nantong & 3.95 \\
Changbai mountain & 3.06 \\
Wuyi mountain & 4.33 \\
Liaocheng & 3.51 \\
The SDs & 1.13 \\
\hline
\end{tabular}

triterpenoid is different in varieties of G. lucidum (Liu et al., 1999; Xing and Jiang, 2001; Xing et al., 2004; Zheng et al., 2007). Third, some research suggests that the different drying methods have some effect in the content of polysaccharide of G. lucidum. The own drying for G. lucidum is significantly higher than the direct drying in polysaccharide content, which is considered to be hydrolysis, induced by hydrolytic enzymes (Xing and Jiang, 2001). The content of polysaccharide is higher in asporogenous G. lucidum than in sporiparous G. lucidum. The different parts of G. lucidum have different content of polysaccharide (Shi et al., 2010). Maybe due to the superior cultivation environment in Dabie Mountain, the triterpenoid content is highest in G. lucidum. There maybe some relations between the high polysaccharide content and the culture medium in Liaocheng where the only G. lucidum were cultured on cotton seed.

\section{CONCLUSION}

This study gives a comprehensive assessment of the G. Lucidum in terms of its efficacy and material, it provides shallow datum for the G. Lucidum quality from different areas. The result of the experiment indicated that there was no distinction correlation between polysaccharide and triterpenoid contents. Because of the difference in active ingredient from different origins, we can choose the G. lucidum according to our purposes.

\section{ACKNOWLEDGMENTS}

We thank professor Ding Zimian and his laboratory members for the help of this research, the companies provided the free samples and Beijing Hengjitang Medical Technology Development Co., Ltd.

\footnotetext{
on triterpene constituents Chen, R. Y., and Yu, D. Q. (1990). The and pharmacology of Gano- research advance on the constituents derma lucidum. J. Fungal Res. 2, of Ganoderma lucidum. Yao Xue Xue 68-77.
} 
Chen, Z. L., Wen, L., and Jia, Z. H. (2009). Effect of some trace elements and vitamins on contents of polysaccharide and acid of Ganoderma lucidum. J. Anhui Agric. Sci. 37, 2041-2043.

Ding, P., Qiu, J. Y., Liang, Y. J., and Wang, H. L. (2009). Chromatographic fingerprints of triterpenoid constituents of Ganoderma lucidum. Zhongguo Zhong Yao Za Zhi 34, 2356-2359.

Ding, P., Zeng, Y. E., Lai, X. P., and Xu, H. H. (1999). Effect of places and stages on the contents of Ganoderma lucidum. Chin. Herb. Med. 22, 271-272.

el-Mekkawy, S., Meselhy, M. R., and Nakamura, N. (2007). Anti - HIV1 and anti - HIV-1 protease substances from Ganoderma lucidum. Phytochemistry 49, 1651-1657.

Hou, M. N., and Liu, J. (2010). Ganoderma triterpenoids extraction and determination of the total triterpenoid. Res. Pract. Chin. Med. 24, 70-71.

Huang, Y. J., and Xiao, G. L. (2008). The progress of pharmacology on Ganoderma triterpene. Cuiding J. Tradit. Chin. Med. Pharm. 14, 87-97.

Li, X. H., He, Y. Q., and Li, R. Z. (1997). Determination of content of polysaccharides in different habitats. Zhongguo Zhong Yao Za Zhi 22, 83-84.

Lin, L., Fang, X. H., and Wu, D. (2002). A survey on effective constituents of Ganoderma lucidum. Chin. Tradit. Pat. Med. 24, 293-296.

Lin, Z. B. (2007). Modern Research of Ganoderma. Beijing: Medical University Press.

Liu, C. (2008). Studies on the Chemical Constituents of Ganoderma Sinense \& Ganoderma Tsugae and the
Content Determinations of Triterpene Acids in Ganoderma Specimens, Master's thesis. Institute of Materia Medica, Chinese Academy of Medical Sciences \& Peking Union Medical College. 12.

Liu, Y. P., Cai, H. J., and Lin, L. L. (1999). Determination of polysaccharides in different kinds of culture of Ganoderma lucidum. J. Guangzhou Univ. Tradit. Chin. Med. 1, 54-55.

Lü, C. T., Yao, X. Y., and Sun, C. (2011). Progress of researches on main active substances and pharmacology of Ganoderma Lucidum. J. Anhui Agric. Sci. 17, 50-51.

Min, B. S., Nakamura, N., Miyashiro, H., and Bae, K. W., Hattori, M. (1998). Triterpenes from the spores of Ganoderma lucidum and their inhibitory activity against HIV-1 protease. Chem. Pharm. Bull. 46, 1607-1612.

Morigiwa, A., Kitabatake, K., Fujimoto, Y., and Ikekawa, N. (1986). Angiotensin conv-eating enzymeinhibitory triterpenes from Ganoderma lucidum. Chem. Pharm. Bull. 34, 3025-3028.

Shi, J. Q., Zhang, L. P., Yang, C. Q., and Wang, Y. F. (2010). Study on polysaccharide content in two different variety of Ganoderma lucidum. Chin. J. Exp. Tradit. Med. Formulae 16, 104-106.

Wang, H. C., and Sun, S. Y. (1990). Analysis on the amino acids and trace elements in wild and artificial cultivation Ganoderma lucidum collected from Taishan. Tradit. Chin. Med. Pat. Prescript. 12, 14.

Wei, H. P., Li, X. G., Pu, S. C., Liu, Z. Y., and Liu, Y. Q. (2006). Changing tendency of ganoderan content in Ganoderma lucidum during fungus growth. Guiding J. Tradit. Chin. Med. Pharm. 34, 10-12.
Xing, Z. T., and Jiang, H. H. (2001). Comparative study on polysaccharide contents in different Ganoderma Species. Edible Fungi 6, 4-5.

Xing, Z. T., Yu, Q. H., Zhang, J. S., and Pan, Y. (2004). Comparative study on triterpenes in different Ganoderma Species. Chin. Herb. Med. 27, 575-576.

Xu, L. C., and Xu, C. S. (2003). Determination of content of polysaccharides of 18 varieties of Ganoderma lucidum karst with different origins. World Sci. Technol. Mod. Tradit. Chin. Med. Mater. Med. 6, 57-60.

Yang, Y. Z., Su, Q. H., and Dong, D. C. (1995). Fruiting bodies of Ganoderma triterpenoids for mice exclude the effect of acute liver disorders. Beijing Da Xue Xue Bao 24, 50-53.

Ye, M. F., Qin, Y. R., Liu, X. H., and Qin, B. S. (2010). Comparative analysis of Ganoderma lucidum polysaccharide content in several wild and artificial cultivation Ganoderma lucidum collected from northwest of Guangxi. Zhong Cao Yao 14, 186-188.

Zhang, X. Y., and Yang, C. Q. (2006). Chemical constituents and pharmacological activities of Ganoderma lucidum. Foreign Medical Sciences 21 , 152-155.

Zhao, J., Chen, X. H., and Bi, K. S. (2009). Simultaneous HPLC determination of four triterpenoid acids in Ganoderma lucidum. China Journal of Chinese Materia Medica 34, 2220-2222.

Zhao, D. X., Yang, X. L., Wang, B., and Zhu, H. (1999). Certain progress in the study of Ganoderma Lucidum. Acta Edulis Fungi 6, 59-64.

Zhao, M. W., Wang, C. G., Bao, P., Xing, Z. T., Liang, W. Q., Yu,
J. Q., Shang, X. D., Men, D. Y., Wang, N., and Pan, Y. J. (2002). Triterpenes and polysaccharide contents of liquid-culturing mycelium of different Ganoderma lucidum. Edible Fungi China 22, 43-46.

Zheng, L. Y., Huang, X. Q., Zen, J., Xu, X. Y., Jiang, N., and Luo, X. (2007) Compared and analysed polysaccharides and triterpene content of different Ganoderma strains. Sichuan Da Xue Xue Bao Yi Xue Ban 44 1121-1124.

Zhou, C. Y., Tang, Q. J., Yang, Y., Guo, Q., Bai, Y. Q., and Pan, Y. J. (2004). Antitumor effect of ganoderic acid from Ganoderma lucidum. Mycosystema 23, 275-279.

Conflict of Interest Statement: The authors declare that the research was conducted in the absence of any commercial or financial relationships that could be construed as a potential conflict of interest.

Received: 19 December 2011; accepted: 19 March 2012; published online: 09 April 2012.

Citation: Lu J, Qin J-Z, Chen P, Chen $X$, Zhang Y-Z and Zhao S-J (2012) Quality difference study of six varieties of Ganoderma lucidum with different origins. Front. Pharmacol. 3:57. doi: 10.3389/fphar.2012.00057

This article was submitted to Frontiers in Ethnopharmacology, a specialty of Frontiers in Pharmacology.

Copyright (c) 2012 Lu, Qin, Chen, Chen, Zhang and Zhao. This is an open-access article distributed under the terms of the Creative Commons Attribution Non Commercial License, which permits noncommercial use, distribution, and reproduction in other forums, provided the original authors and source are credited. 